\title{
A snapshot of genetic lineages of Mycobacterium tuberculosis in Ireland over a two-year period, 2010 and 2011
}

M M Fitzgibbon (MFitzgibbon@STJAMES.IE) ${ }^{1}$, N Gibbons ${ }^{1}$, E Roycroft' ${ }^{1,2}$, S Jackson ${ }^{3}$, J O’Donnell ${ }^{3}$, D 0’Flanagan ${ }^{3}$, T R Rogers ${ }^{1,2}$

1. Irish Mycobacteria Reference Laboratory, St. James' Hospital, Dublin, Ireland

2. Department of Clinical Microbiology, Trinity College, Dublin, Ireland

3. Health Protection Surveillance Centre, Dublin, Ireland

Citation style for this article:

Fitzgibbon MM, Gibbons N, Roycroft E, Jackson S, O'Donnell J, O'Flanagan D, Rogers TR. A snapshot of genetic lineages of Mycobacterium tuberculosis in Ireland over a two-year period, 2010 and 2011. Euro Surveill. 2013;18(3):pii=20367. Available online: http://www.eurosurveillance.org/ViewArticle.aspx?Articleld=20367

Mycobacterial interspersed repetitive-unit-variablenumber tandem repeat typing alone was used to investigate the genetic lineages among 361 Mycobacterium tuberculosis strains circulating in Ireland over a twoyear period, 2010 and 2011 . The majority of isolates, $63 \%$ (229/361), belonged to lineage 4 (Euro-American), while lineages 1 (Indo-Oceanic), 2 (East-Asian) and 3 (East-African-Indian) represented $12 \%$ of isolates each (42/361, 45/361, and 45/361, respectively). Sub-lineages Beijing (lineage 2), East-African-Indian (lineage 1) and Delhi/central-Asian (lineage 3) predominated among foreign-born cases, while a higher proportion of Euro-American lineages were identified among cases born in Ireland. Eighteen molecular clusters involving 63 tuberculosis (TB) cases were identified across four sub-lineages of lineage 4 . While the mean cluster size was 3.5 TB cases, the largest cluster (involving 12 Irish-born cases) was identified in the Latin American-Mediterranean sub-lineage. Clustering of isolates was higher among Irish-born TB cases (47 of 63 clustered cases), whereas only one cluster (3/63) involved solely foreign-born individuals. Four multidrug-resistant cases identified during this period represented lineages 2 and 4. This study provides the first insight into the structure of the $M$. tuberculosis population in Ireland.

\section{Introduction}

Tuberculosis (TB) caused by Mycobacterium tuberculosis remains a serious challenge to public health worldwide. Despite an overall decline in case notification rates for TB across Europe, rates vary significantly, with the highest rates reported from eastern Europe and the Baltic States [1]. Multidrug-resistant (MDR) TB continues to be a major problem and an added burden in highincidence countries such as Romania (108.2/100,000) and the Baltic states Lithuania $(62.1 / 100,000)$, Latvia $(43.2 / 100,000)$ and Estonia $(30.7 / 100,000)$ [1]. Ireland has a low incidence, with notification rates that ranged between 9.7 and 11.3 per 100,000 population between 2001 and 2010 although to some extent this may have been influenced by migrants arriving from high-burden countries [1-2]. In Ireland, TB is a statutorily notifiable disease, and in a recent report on the epidemiology of TB in the country, the proportion of culture-confirmed TB cases was $71.2 \%$ in 2009 and $63.2 \%$ in 2010 (data from 2010 were not finalised at the time of submission) [3]. These proportions are similar to those reported for previous years [3].

Disruption of transmission chains is a key factor in controlling TB both at a national and international level [4]. In recent years, there have been significant advances in developing the molecular tools required for rapid diagnosis of TB [4]. Analysis of variable-number tandem repeat (VNTR) sequences at mycobacterial interspersed repetitive units (MIRU) has emerged as a valuable marker for genotyping strains of the $M$. tuberculosis complex [5]. In large population-based studies, MIRU-VNTR typing has been shown to have similar discriminatory power when compared to IS6110 restriction fragment length polymorphism (RFLP) typing [5-7]. An optimised set of 24 MIRU-VNTR markers has become the gold standard for genotyping $M$. tuberculosis complex strains worldwide [5,7]. MIRU-VNTR typing is a PCRbased method that yields rapid, reproducible results that are expressed as a 24-digit numerical code which allows for easy exchange of data [5-8]. This method can be applied to early mycobacterial cultures and more recently has been successfully applied directly to smear-positive specimens [5,9]. Previous studies have shown MIRU-VNTR typing to be useful in comparing strains (i) at national and international level, (ii) among household contacts, (iii) associated with drug resistance and (iv) to determine the evolutionary pathway of TB [5-6,10-16]. At European level, MIRU-VNTR typing has been adopted for the molecular surveillance of the international transmission of MDR-TB and extensively drug-resistant TB [7].

In November 2009, molecular genotyping in the form of 24-locus MIRU-VNTR typing was introduced at the 
Irish Mycobacteria Reference Laboratory (IMRL) which both cultures and receives isolates from microbiology laboratories around the country. To allow for rapid, high-throughput genotyping of $M$. tuberculosis, the commercial MIRU-VNTR typing kit (GenoScreen, Lille, France) was introduced in 2010 [5,7]. As 24-locus MIRUVNTR typing is considered the gold standard genotyping method, all $M$. tuberculosis isolates identified at the IMRL are currently typed prospectively with this method, and it is envisaged that all $M$. tuberculosis isolates recovered since 2000 will be typed on a retrospective basis.

Here we report the first analysis of the structure of the M. tuberculosis population in Ireland for isolates recovered during 2010 and 2011 following the introduction of 24 -locus MIRU-VNTR typing to the diagnostic laboratory. It needs to be noted that at the time there was an under-representation of isolates from the southern region of Ireland.

\section{Methods}

\section{MIRU-VNTR typing}

$M$. tuberculosis isolates $(n=361)$ recovered in or referred to the IMRL over a two-year period (2010-11) were typed with the MIRU-VNTR typing kit (GenoScreen) [5]. Validation of the MIRU-VNTR technique was performed using the MIRU-VNTR Calibration Kit (GenoScreen). PCR products were subjected to electrophoresis using a 3130 genetic analyser (Applied Biosystems). Sizing of fragments and MIRU-VNTR allele assignation was performed using GeneMapper software (Applied Biosystems). Phylogenetic lineages were assigned to each isolate using the MIRU-VNTRplus online tool [17-18].

The 24-locus MIRU-VNTR panel comprised the following loci: MIRU 02, VNTR 42, VNTR 43, MIRU 04, MIRU 40, MIRU 10, MIRU 16, VNTR 1955, MIRU 20, QUB 11b, ETR A, VNTR 46, VNTR 47, VNTR 48, MIRU 23, MIRU 24, MIRU 26, MIRU 27, VNTR 49, MIRU 31, VNTR 52, QUB 26, VNTR 53, and MIRU 39. The MIRU-VNTR profiles are reported as a series of 24 numbers that correspond to the number of alleles at each of the loci described above.

Clusters of isolates were defined as two or more isolates with indistinguishable MIRU-VNTR patterns. The strain clustering rate was calculated as $\left(n_{c}-c\right) / n$, where $\mathrm{n}_{\mathrm{c}}$ was the total number of strain-clustered cases, $c$ was the number of clusters and $n$ was the total number of isolates [5].

\section{Epidemiological analysis}

Enhanced surveillance of TB was implemented in Ireland in 1998. Enhanced TB notification forms are completed by public health doctors, summarising all available clinical, microbiological, histological and epidemiological information. These data are collated in the regional public health departments. Anonymised data are then submitted electronically to the Health Protection Surveillance Centre (HPSC) for the production of reports on a weekly, quarterly and annual basis.

Since January 2011, cases of TB have been reported through the Computerised Infectious Disease Reporting system (CIDR). CIDR is a web-based system developed to integrate case-based clinical and laboratory data in order to manage the surveillance and control of notifiable infectious diseases in Ireland. Prior to using CIDR for TB surveillance, MIRU-VNTR typing results were not linked to case-based epidemiological data. In addition to recording sporadic case-based data, CIDR also facilitates the reporting of clustered cases, according to Irish outbreak case definitions [2]. Clustered cases can be reported via a summary aggregate outbreak data module to which the relevant disaggregate case-based surveillance data can also be linked.

\section{Data analysis}

The TB enhanced surveillance data for 2011 (epidemiological and linked laboratory data) used in this publication were extracted from CIDR on 17 April 2012 using Business Objects XI software and were analysed using Microsoft Excel. Data for 2011 were provisional at the time of extraction and subject to ongoing validation and revision.

\section{Results}

Results are presented in two separate sections. In the first part, genotyping results for isolates recovered in 2010-11 are presented. As epidemiological data linking was available from 2011 onwards, the second part of the results section (enhanced surveillance) refers to genotyping results linked to epidemiological data for 2011 isolates only.

\section{Mycobacterium tuberculosis genotyping, 2010-11}

Some $361 M$. tuberculosis isolates were recovered in or referred to the IMRL during 2010-11, representing $63.6 \%$ of culture-positive cases identified through the national TB surveillance system in that period. Genotyping of $M$. tuberculosis isolates recovered during the study period yielded four global lineages (Table 1$)$. The majority $(63 \%)$ belonged to lineage 4 (Euro-American), while lineages 1 (Indo-Oceanic), 2 (East-Asian) and 3 (East-African-Indian) represented $12 \%$ each. Among the 229 Euro-American strains, sublineages Latin American-Mediterranean (LAM) (23\%), Haarlem (21\%), H37Rv (19\%) and Haarlem/X (13\%) were most prevalent (Table 1).

Within lineage 4,18 clusters were identified involving 63 TB cases (Table 2). The strain clustering rate varied between different sub-lineages, but was highest for the LAM sub-lineage $(6.9 \%)$. While the mean cluster size was 3.5 TB cases, the largest cluster (involving 12 Irish-born cases, representing $19 \%$ of all clustered isolates) was identified within the LAM sub-lineage (Table 
TABLE 1

Distribution of lineages among Mycobacterium

tuberculosis isolates, Ireland, 2010-11 $(n=361)$

\begin{tabular}{|c|c|c|c|}
\hline Global lineage & Sub-lineage & $\begin{array}{l}\text { No. of } \\
\text { isolates }\end{array}$ & $\begin{array}{l}\% \text { of } \\
\text { isolates }\end{array}$ \\
\hline 1 Indo-Oceanic & East-African-Indian & 42 & 12 \\
\hline 2 East-Asian & Beijing & 45 & 12 \\
\hline 3 East-African-Indian & Delhi/central-Asian & 45 & 12 \\
\hline \multirow[t]{14}{*}{4 Euro-American } & Lineage 4 total & 229 & 63 \\
\hline & $\begin{array}{l}\text { Latin American- } \\
\text { Mediterranean }\end{array}$ & 52 & \\
\hline & Haarlem & 47 & \\
\hline & $\mathrm{H}_{37} \mathrm{Rv}$ & 44 & \\
\hline & Haarlem/X & 29 & \\
\hline & Cameroon & 13 & \\
\hline & $S$ & 6 & \\
\hline & TUR & 8 & \\
\hline & $\mathrm{X}$ & 5 & \\
\hline & Ghana & 3 & \\
\hline & URAL & 3 & \\
\hline & Uganda I \& II & 6 & \\
\hline & NEW-1 & 2 & \\
\hline & Others $^{a}$ & 11 & \\
\hline Total & & 361 & 100 \\
\hline
\end{tabular}

${ }^{a}$ The category Others includes isolates for which the sub-lineages were not clearly defined

2). Four other clusters within the LAM sub-lineage contained between $4.7 \%(3 / 63)$ and $12.7 \%(8 / 63)$ of clustered cases. Among the clustered cases of Haarlem, $\mathrm{H} 37 \mathrm{Rv}$ and Haarlem/X, cluster sizes ranged from 3.2 to $9.5 \%$, at $3.2 \%$ and from 3.2 to $4.7 \%$ of isolates, respectively.

Only one cluster (3/63) contained exclusively foreignborn individuals, 12 clusters (47/63) involved Irishborn cases only, while five clusters (13/63) were mixed. In addition, one small cluster was observed among the isolates from lineage 2.

The four MDR-TB cases identified during this period represented lineages 2 (Beijing) and 4 (Ural, $\mathrm{H}_{37 \mathrm{Rv}}$ and LAM). None of the MDR-TB cases were clustered.

Tuberculosis enhanced surveillance data for 2011 isolates (epidemiological and laboratory) In 2011, 432 TB cases were provisionally reported on CIDR, of which approximately $166(38 \%)$ were typed. At the time of data extraction, 136 TB cases were updated to include MIRU typing results (representing $81.9 \%$ of 166 typed isolates). Of the 136 TB cases with a MIRU typing result, 34 were clustered in 11 clusters with different MIRU types. Clusters ranged in size from eight to two TB cases. Of the 11 MIRU type clusters, five, comprising 18 TB cases, were confirmed by public health departments as outbreaks meeting the Irish case definition.

The Beijing sub-lineage was most prevalent (15.4\%) and associated with a small cluster. Sub-lineages Haarlem, LAM, and $\mathrm{H}_{37} \mathrm{Rv}$ were most prevalent among lineage 4 strains, while lineage 1 and lineage 3 represented $11.8 \%$ and $10.3 \%$ of typed isolates, respectively. Interestingly, isolates recovered from pulmonary specimens were mostly correlated with lineage 4 strains, while the majority of isolates recovered from extra-pulmonary specimens belonged to lineages 1 and 3 (Figure 1). In lineage 3, nine of 14 isolates were recovered from patients born in Pakistan, while the remaining five isolates were recovered from patients born in India $(n=2)$, Kenya $(n=1)$, Nepal $(n=1)$ and Nigeria $(n=1)$. Only one lineage 1 isolate was recovered from an Irishborn patient, while six were recovered from patients born in the Philippines. Other countries represented among lineage 1 isolates were Bangladesh, India, Mozambique, Pakistan, Somalia and Vietnam.

The distribution of lineages among Irish-born and foreign-born TB cases is shown in Figure 2. Of the 127 TB cases for whom MIRU-VNTR and country of birth were known, $51.5 \%$ were foreign-born and $41 \%$ were Irishborn. Lineages 1, 2 and 3 predominated among foreignborn TB cases, while a higher proportion of lineage 4 isolates were identified among Irish-born cases.

\section{Discussion}

This study has provided a snapshot of the genetic diversity of $M$. tuberculosis in Ireland. Due to the small numbers of isolates in our study, statistical analysis would not be significant and was not performed. Although data on sub-lineages were analysed by age and sex, the resulting frequencies were too small to draw firm conclusions from. However, when age and sex analyses were further stratified by country of birth, these data were broadly similar to the age and sex profile of the Irish TB notification data.

A large diverse group of isolates has been identified, suggesting a low degree of active transmission among TB patients. The distribution of genetic lineages is similar to other recent studies that used different typing techniques and in which lineage 4 (Euro-American) predominated among circulating $M$. tuberculosis strains [12,19-21]. In previous work conducted in the southwest region of Ireland, lineage 4 predominated, and clustering of isolates was associated with Irish nationals and lineage 4 isolates only [22]. In our study, the distribution of genetic lineages among extra-pulmonary specimens (where lineages 1 and 3 predominated) was similar to a recently published large-scale study conducted in the United States (US) investigating the relationship between genetic lineages and clinical sites of infection [23]. In the US study, the highest percentage of isolates recovered from extra-pulmonary 


\begin{tabular}{|c|c|c|c|c|c|c|}
\hline Sub-lineage & $\begin{array}{l}\text { Total no. } \\
\text { of cases }\end{array}$ & $\begin{array}{l}\text { No. of } \\
\text { clustered } \\
\text { cases (\%) }\end{array}$ & $\begin{array}{l}\text { No. of } \\
\text { clusters }\end{array}$ & $\begin{array}{l}\text { Strain } \\
\text { clustering } \\
\text { rate (\%) }\end{array}$ & $\begin{array}{l}\text { No. of isolates/ } \\
\text { cluster (\% } \\
\text { clustered cases) }\end{array}$ & MIRU-VNTR profile \\
\hline \multirow{5}{*}{ Latin American-Mediterranean } & \multirow{5}{*}{52} & \multirow{5}{*}{$30(8.3)$} & \multirow{5}{*}{5} & \multirow{5}{*}{6.9} & $3(4.7)$ & 124244332224126153332832 \\
\hline & & & & & $8(12.7)$ & 142244332224126153322622 \\
\hline & & & & & $12(19)$ & 142244332224126143322622 \\
\hline & & & & & $3(4.7)$ & 132244332224125153322222 \\
\hline & & & & & $4(6.3)$ & 132244332224126133322622 \\
\hline \multirow{6}{*}{ Haarlem } & \multirow{6}{*}{47} & \multirow{6}{*}{$18(5)$} & \multirow{6}{*}{6} & \multirow{6}{*}{$3 \cdot 3$} & $2(3.2)$ & 223225342334425143323332 \\
\hline & & & & & $6(9.5)$ & 223225342334425153323 32 \\
\hline & & & & & $2(3.2)$ & 123235332634425143423332 \\
\hline & & & & & $4(6.3)$ & 223225342334425143323 32 \\
\hline & & & & & $2(3.2)$ & 223235331532423153333632 \\
\hline & & & & & $2(3.2)$ & 223215332334425153323632 \\
\hline \multirow{4}{*}{$\mathrm{H}_{37} \mathrm{Rv}$} & \multirow{4}{*}{44} & \multirow{4}{*}{$8(2.2)$} & \multirow{4}{*}{4} & \multirow{4}{*}{1.1} & $2(3.2)$ & 224243122234225153234422 \\
\hline & & & & & $2(3.2)$ & 224243122434225153335512 \\
\hline & & & & & $2(3.2)$ & 2242132225342261533334522 \\
\hline & & & & & $2(3.2)$ & 224213322334226153335522 \\
\hline \multirow{3}{*}{ Haarlem/X } & \multirow{3}{*}{29} & \multirow{3}{*}{$7(1.9)$} & \multirow{3}{*}{3} & \multirow{3}{*}{1.1} & $2(3.2)$ & 224234342334425154135832 \\
\hline & & & & & $3(4.7)$ & $243244332434425153343 a 32$ \\
\hline & & & & & $2(3.2)$ & 243234332234425143331832 \\
\hline Total clustered lineage 4 cases & 172 & 63 & 18 & - & - & - \\
\hline
\end{tabular}

a The numbers in this 24-digit profile correspond to the number of alleles at each of the following loci: MIRU 02, VNTR 42, VNTR 43, MIRU 04, MIRU 40, MIRU 10, MIRU 16, VNTR 1955, MIRU 20, QUB 11b, ETR A, VNTR 46, VNTR 47, VNTR 48, MIRU 23 , MIRU 24 , MIRU 26, MIRU 27, VNTR 49, MIRU 31, VNTR 52, QUB 26, VNTR 53, MIRU 39.

specimens was from lineages 1 (22.6\%) and 3 (34.3\%) [23]. However, due to the small numbers of exclusive extra-pulmonary specimens $(n=30)$ and limited epidemiological data, statistical analysis of the relationship between lineage and clinical site of infection was not possible in our report.

Molecular clustering of isolates in our study was more common among Irish-born individuals. These findings were similar to a previous Irish study conducted by Ojo et al. in the south-west region of Ireland, but unlike a recent study conducted in Switzerland $[12,22]$. We identified 18 clusters in lineage 4 , and the mean cluster size was 3.5 TB cases. The largest cluster, involving 12 TB cases, belonged to the LAM lineage and spanned a period of 18 months. A second cluster identified in the LAM lineage differed by a single locus variant (SLV) at locus 2996. Similarly, in the Haarlem lineage, the two largest clusters differed by a SLV at locus 2996 also. MIRU 26 (or locus 2996) has yielded stable comparable results in a large-scale study investigating 824 M. tuberculosis isolates conducted at the Institut Pasteur de Lille, France, in 2006 [5]. Molecular typing played a key role in identifying a dominant $M$. tuberculosis strain (known as the Mercian strain) circulating in the West Midlands region in the United Kingdom (UK) over a five-year period, highlighting the importance of cluster analysis [14]. Prospective molecular typing can identify rapidly expanding clusters of $M$. tuberculosis before they spread further into the community. A single dominant MIRU-VNTR type was not observed in this study, however, this could be due to the study period being short. In contrast, prospective molecular typing of $M$. tuberculosis by RFLP, performed since 1993 in the Netherlands, has proven to be effective. DNA fingerprinting data has been shown to be a powerful tool in defining epidemiological links and guiding TB control programmes in the Netherlands [24-25].

Another limitation of this study is that only one typing method was used to investigate the $M$. tuberculosis population structure in Ireland. Previous studies have shown that a combination of MIRU-VNTR typing and spoligotyping can differentiate more readily between M. tuberculosis strains [26-27]. However, in a previous Irish study using both spoligotyping and MIRU-VNTR typing, MIRU-VNTR typing identified clusters among spoligotype groups, thus providing supporting evidence that MIRU-VNTR typing is a more discriminatory typing method [22]. The discriminatory power of the 24-locus MIRU-VNTR panel used in this study has shown to be similar to IS6110 RFLP analysis [5]. However, the discriminatory power of 24-locus MIRU-VNTR typing differs among genetic lineages, and the inclusion of 


\section{FIGURE 1}

Distribution of Mycobacterium tuberculosis lineages by site of infection, Ireland, 2011 (n=136)

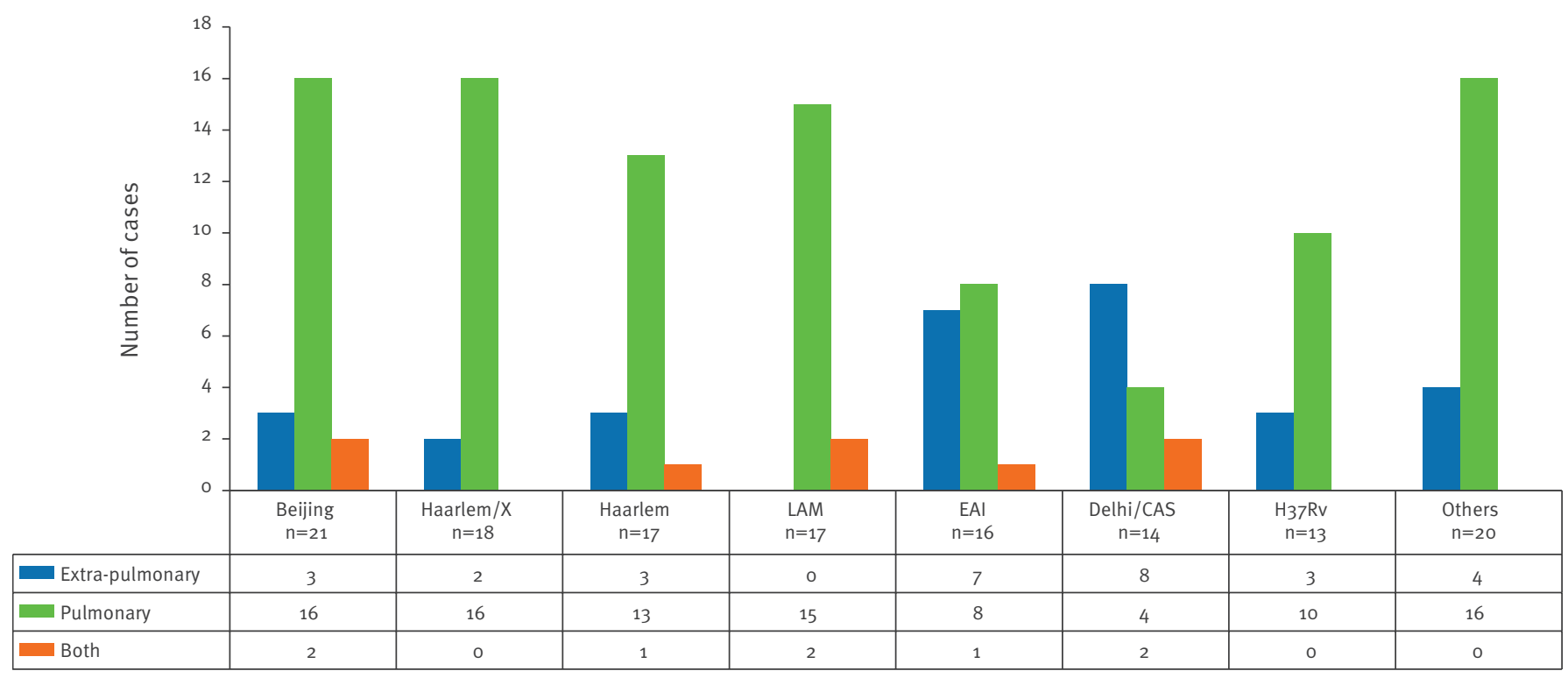

CAS: central-Asian; EAI: east-African-Indian; LAM: Latin American-Mediterranean.

\section{FIGURE 2}

Distribution of lineages among Irish and non-Irish typed Mycobacterium tuberculosis cases, Ireland, 2011 (n=127)

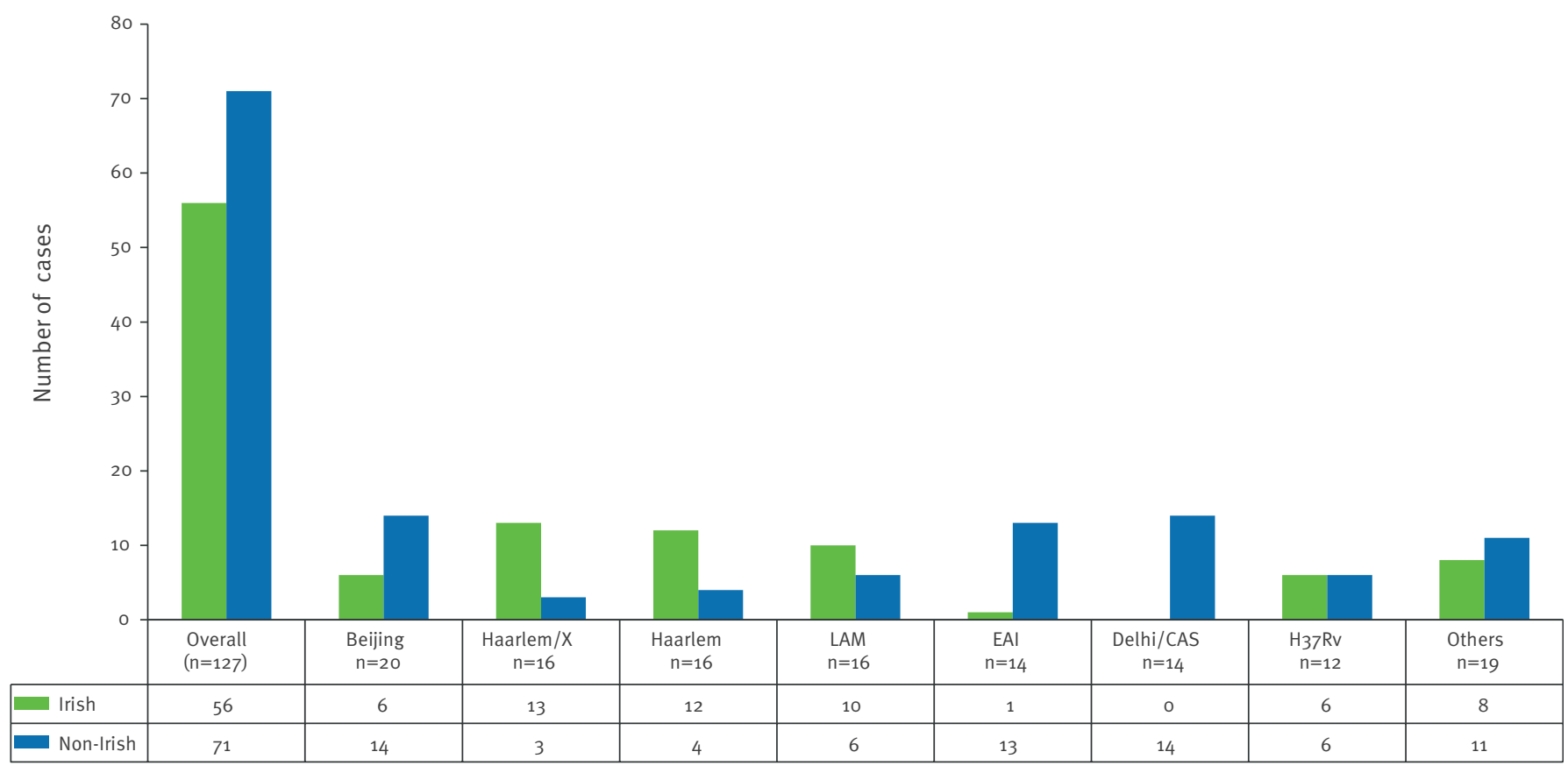

CAS: central-Asian; EAI: east-African-Indian; LAM: Latin American-Mediterranean. 
additional hypervariable loci may be required to differentiate among strains of lineages 2 (Beijing) and 3 (Delhi/central-Asian). For enhanced cluster or outbreak analysis, whole-genome sequencing has been shown to differentiate among strains with identical 24-locus MIRU-VNTR patterns [28-29]. The role of whole-genome sequencing in investigating community outbreaks in the UK was reported recently [29]. Walker et al. estimated that the rate of genetic changes was 0.5 single nucleotide polymorphisms (SNPs) per genome per year. Furthermore, the maximum number of genetic changes over three years would be five SNPs and 10 SNPs over 10 years [29]. It has also been proposed that clustering of isolates increases over longer periods as transmission chains are more efficiently analysed and reported [30]. But the M. tuberculosis genotype involved in the cluster must be considered as for example the Beijing lineage has increased ability to spread and cause disease. While clustering was limited in our study, the study period was too short to draw clear conclusions.

Although the reproducibility of MIRU-VNTR typing has been well documented, results from the first worldwide proficiency study on this method were surprising [7]. Intra- and inter- laboratory reproducibility varied depending on the typing methods employed in each laboratory. In our setting, when the commercial MIRUVNTR typing kit was used to analyse the quality control panel, $100 \%$ concordance was achieved with the reference data (30/30 tested strains) and 100\% intra-laboratory reproducibility was achieved. These findings are important to consider when typing data is exchanged between laboratories.

Although six of the 11 MIRU typing clusters identified during 2011 were not confirmed as outbreaks by public health departments, it is possible that the reason why four of these clusters did not meet the Irish TB outbreak case definitions was the small number $(n=2)$ of involved cases [2].

In summary, this study has provided the first insights into the structure of the $M$. tuberculosis population in Ireland. Although the incidence of TB has remained static in Ireland over the last decade, there has been mass immigration to this island nation. Not surprisingly, lineage 4 predominated among circulating strains of $M$. tuberculosis in the present study. But the degree of diversity among $M$. tuberculosis was unexpected. Future studies in the IMRL involving retrospective genotyping analysis of $M$. tuberculosis isolates collected since 2000 may provide an interesting epidemiological picture. Continued molecular surveillance is important as it has been suggested that the transmissibility profile of $M$. tuberculosis strains may be influenced by their genetic and evolutionary background. This understanding of the dynamics of $M$. tuberculosis strains will provide novel insights into the $M$. tuberculosis population structure and how it relates to the epidemiology of TB in Europe and beyond.
Acknowledgments

The authors would like to thank all the departments of public health, clinicians and laboratories for providing the surveillance data on these TB cases.

\section{References}

1. European Centre for Disease Prevention and Control (ECDC). Annual Epidemiological Report 2011. Reporting on 2009 surveillance data and 2010 epidemic intelligence data. Stockholm: ECDC; 2011. Available from: http://www.ecdc. europa.eu/en/publications/Publications/Forms/ECDC_ DispForm.aspx?ID $=767$

2. Health Protection Surveillance Centre (HPSC). Guidelines on the Prevention and Control of Tuberculosis in Ireland 2010. Dublin: HPSC; 2010. Available from: http://www.hpsc.ie/hpsc/ AboutHPSC/ScientificCommittees/Publications/File,4349,en. pdf

3. Health Protection Surveillance Centre (HPSC). Report on the Epidemiology of TB in Ireland, 2009. Dublin: HPSC, 2012. [Accessed 17 Jan 2013]. Available from: http://www.ndsc.ie/ hpsc/A-Z/VaccinePreventable/TuberculosisTB/Epidemiology/ SurveillanceReports/2009/File,13261,en.pdf

4. Barnes PF. Cave MD. Molecular epidemiology of tuberculosis. New Engl J Med. 2003;349(12):1149-56.

5. Supply P, Allix C, Lesjean S, Cardoso-Oelemann M, RüschGerdes S, Willery E, et al. Proposal for standardization of optimized mycobacterial interspersed repetitive unit-variable number tandem repeat typing of Mycobacterium tuberculosis. Clin Microbiol. 2006;44(12):4498-510.

6. Bidovec-Stojkovic U, Zolnir-Dovc M, Supply P. One year nationwide evaluation of 24-locus MIRU-VNTR genotyping on Slovenian Mycobacterium tuberculosis isolates. Respir Med. 2011;105 Suppl 1:S67-73.

7. de Beer J, Kremer K, Ködmön C, Supply P, van Soolingen D, Global Network for the Molecular Surveillance of Tuberculosis 2009. First worldwide proficiency study on variable-number tandem-repeat typing of Mycobacterium tuberculosis complex strains. J Clin Microbiol. 2012;50(3):662-9.

8. Kanduma E, McHugh TD, Gillespie SH. Molecular methods for Mycobacterium tuberculosis strain typing: a user's guide. J Appl Microbiol. 2003;94(5):781-91.

9. Alonso M, Herranz M, Martinez Lirola M, Gonzalez-Rivera M, Bouza E, Garcia de Viedma D. Real-time molecular epidemiology of tuberculosis by direct genotyping of smearpositive clinical specimens. J Clin Microbiol. 2012;50(5):1755-7.

10. Augustynowicz-Kopeć E, Jagielski T, Kozińska M, Kremer K, Van Soolingen D, Bielecki J, et al. Transmission of tuberculosis within family-households. J Infect. 2012;64(6):596-608.

11. Prodinger WM, Polanecký V, Kozáková B, Müllerová M, Mezenský L, Kaustová J, et al. Molecular epidemiology of tuberculosis in the Czech Republic, 2004: analysis of $M$. tuberculosis complex isolates originating from the city of Prague, south Moravia and the Moravian-Silesian region. Cent Eur J Public Health. 2006;14(4):168-74.

12. Fenner L, Gagneux S, Helbling P, Battegay M, Rieder HL, Pfyffer GE, et al. Mycobacterium tuberculosis transmission in a country with low incidence: role of immigration and HIV infection. J Clin Microbiol. 2012;50(2):388-95.

13. Maguire H, Brailsford S, Carless J, Yates M, Altass L, Yates S, et al. Large outbreak of isoniazid-monoresistant tuberculosis in London, 1995 to 2006: case-control study and recommendations. Euro Surveill. 2011;16(13):pii=19830. Available from: http://www.eurosurveillance.org/Viewarticle. aspx?Articleld $=19830$

14. Evans JT, Serafino Wani RL, Anderson L, Gibson AL, Grace Smith E, Wood A, et al. A geographically-restricted but prevalent Mycobacterium tuberculosis strain identified in the West Midlands region of the UK between 1995 and 2008. PLoS One. 2011; 6(3):e17930.

15. Wirth T, Hildebrand F, Allix-Béguec C, Wölbeling F, Kubica $\mathrm{T}$, Kremer K, et al. Origin, spread and demography of the Mycobacterium tuberculosis complex. PLoS Pathog. 2008;4(9):e1000160.

16. Sails AD, Barrett A, Sarginson S, Magee JG, Maynard P, Hafeez I, et al. Molecular epidemiology of Mycobacterium tuberculosis in East Lancashire 2001-2009. Thorax. 2011;66(8):709-13.

17. Weniger T, Krawczyk J, Supply P, Niemann S, Harmsen D. MIRU-VNTRplus: a web tool for polyphasic genotyping of Mycobacterium tuberculosis complex bacteria. Nucleic Acids Res. 2010;38:W326-31.

18. Allix-Béguec C, Harmsen D, Weniger T, Supply P, Niemann S. Evaluation and strategy for use of MIRU-VNTRplus, a 
multifunctional database for online analysis of genotyping data and phylogenetic identification of Mycobacterium tuberculosis complex isolates. J Clin Microbiol. 2008;46(8):2692-9.

19. Roetzer A, Sieglinde S, Diel R, Gasau F, Ubben T, di Nauta A, et al. Evaluation of Mycobacterium tuberculosis typing methods in a 4-year study in Schleswig-Holstein, Northern Germany. J Clin Microbiol. 2011;49(12):4173-8.

20. Brudey K, Driscoll JR, Rigouts L, Prodinger WM, Gori A, AlHajoj SA, et al. Mycobacterium tuberculosis complex genetic diversity: mining the fourth international spoligotyping database (SpolDB4) for classification, population genetics and epidemiology. BMC Microbiol. 2006;6:23.

21. Homolka S, Post E, Oberhauser B, George AG, Westman L, Dafae D, et al. High genetic diversity among Mycobacterium tuberculosis complex strains from Sierra Leone. BMC Microbiol. 2008;8:103.

22. Ojo 00, Sheehan S, Corcoran D, Nikolayevsky V, Brown T, O'Sullivan M, et al. Molecular epidemiology of Mycobacterium tuberculosis isolates in Southwest Ireland. Infect Genet Evol. 2010;10(7):1110-6.

23. Click ES, Moonan PK, Winston CA, Cowan LS, Oeltmann JE. Relationship between Mycobacterium tuberculosis phylogenetic lineage and clinical site of tuberculosis. Clin Infect Dis. 2012;54(2):211-9.

24. Borgdorff MW, Nagelkerke NJD, de Haas PEW, van Soolingen D. Transmission of Mycobacterium tuberculosis depending on the age and sex of source cases. Am J Epidemiol. 2001;154(10):934-43.

25. Lambregts-van Weezenbeek CS, Sebek MM, van Gerven PJ, de Vries G, Verver S, et al. Tuberculosis contact investigation and DNA fingerprint surveillance in The Netherlands: 6 years' experience with nation-wide cluster feedback and cluster monitoring. Int J Tuberc Lung Dis. 2003;7(12 Suppl 3):S463-70.

26. Oelemann MC, Diel R, Vatin V, Haas W, Rüsch-Gerdes S, Locht C, et al. Assessment of an optimized mycobacterial interspersed repetitive-unit-variable number of tandem-repeat typing system combined with spoligotyping for populationbased molecular epidemiology studies of tuberculosis. J Clin Microbiol. 2007;45(3):691-7.

27. Valcheva V, Mokrousov I, Narvskaya O, Rastogi N, Markova N. Utility of new 24-locus variable-number tandem-repeat typing for discriminating Mycobacterium tuberculosis clinical isolates collected in Bulgaria. J Clin Microbiol. 2008;46(9):3005-11.

28. Gardy JL, Johnston JC, Ho Sui SJ, Cook VJ, Shah L, Brodkin E, et al. Whole-genome sequencing and social-network analysis of a tuberculosis outbreak. N Engl J Med. 2011;364(8):730-9.

29. Walker TM, Ip CLC, Harrell RH, Evans JT, Kapatai G, Dedicoat MJ et al. Whole-genome sequencing to delineate Mycobacterium tuberculosis outbreaks: a retrospective observational study. Lancet Infect Dis. 2012. pii:S1473-3099(12)70277-3. doi: 10.1016/S1473-3099(12)70277-3.

30. Glynn JR, Bauer J, de Boer AS, Borgdorff MW, Fine PE, GodfreyFaussett $P$, et al. Interpreting DNA fingerprint clusters of Mycobacterium tuberculosis. European concerted action on molecular epidemiology and control of tuberculosis. Int J Tuberc Lung Dis. 1999;3(12):1055-60. 